
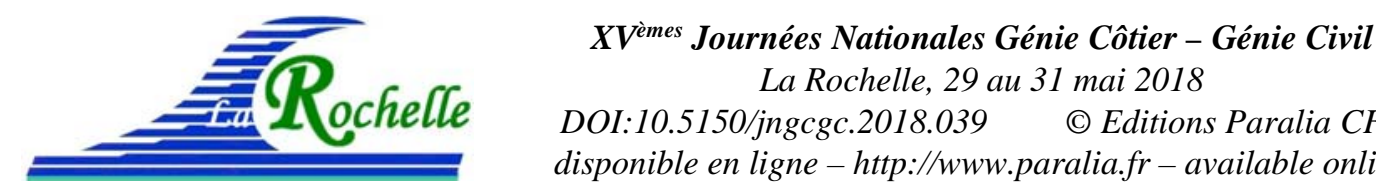

DOI:10.5150/jngcgc.2018.039 C Editions Paralia CFL

disponible en ligne - http://www.paralia.fr - available online

\title{
Suivi vidéo des rejets de dragage du port de plaisance du Brise-Lames au niveau de l'estuaire de l'Adour
}

\author{
Damien DAILLOUX ${ }^{1}$, Clémence FOULQUIER ${ }^{1}$, Didier RIHOUEY ${ }^{1}$ \\ 1. CASAGEC INGENIERIE, 18 Rue Maryse Bastié, 64600 Anglet, France. \\ contact@casagec.fr
}

\section{Résumé :}

Dans le cadre du maintien des cotes d'exploitation du port du Brise-Lames situé sur la rive gauche de l'estuaire de l'Adour, une campagne de dragage a été mise en place entre les mois de Mars et Mai 2016. Les travaux ont été réalisés à l'aide d'une Drague Aspiratrice Stationnaire (DAS) permettant d'extraire les sédiments de manière hydraulique aussi bien dans les zones de navigation du port que sous les pontons et catways sans avoir à démonter ces derniers. Les sédiments dragués ont ensuite été transportés via une canalisation de refoulement et rejetés dans l'Adour. Afin de favoriser la dispersion vers le large, ces rejets ont été réalisés en période de vives eaux établies et lors des courants de jusant, dans un intervalle s'étalant d'une heure après la pleine mer $(\mathrm{PM}+1)$ à une heure avant la basse mer $(\mathrm{BM}-1)$, soit des périodes d'intervention de $4 \mathrm{~h}$ en continu.

Conformément à l'arrêté préfectoral, un suivi temps réel des concentrations en matières en suspension (MES) de surface a été mis en place en amont et en aval du port de plaisance via l'installation de deux turbidimètres. Parallèlement, une caméra rotative a été installée au niveau de l'embouchure de l'Adour pour visualiser une zone située à proximité de la station de mesure aval.

La corrélation entre les mesures du turbidimètre aval et les intensités des canaux rouges, verts et bleus (RVB) enregistrés par la caméra a été investiguée dans le but de réaliser un suivi des concentrations en MES en surface. La technique repose sur (i) la calibration du turbidimètre à partir de mesures in-situ, (ii) la correction des variations d'intensité lumineuse susceptibles de perturber la mesure vidéo (correction de la balance des blancs) et (iii) l'algorithme de corrélation de la forme [MES] = $\mathrm{f}$ (RVB corrigés).

Les résultats montrent une bonne corrélation et l'outil vidéo a permis de dresser un bilan des concentrations en MES sur des échelles spatiotemporelles plus exhaustives. La dynamique des MES de surface a ainsi pu être mise au regard des activités de dragage et des apports de la rivière.

\section{Mots-clés :}

Suivi vidéo, Rejet de dragage, Matière en suspension, Estuaire. 


\section{Thème 3 - Instrumentation, mesures, imagerie et télédétection}

\section{Introduction}

Situé sur la commune d'Anglet dans le département des Pyrénées Atlantique, le port de plaisance du Brise-Lames est situé en rive gauche de l'estuaire de l'Adour. Soumis à un taux d'envasement significatif, le gestionnaire doit régulièrement réaliser des opérations de dragage afin d'entretenir les cotes d'exploitation du port. Pour faire face à cet envasement, des travaux ont été réalisé à l'aide d'une Drague Aspiratrice Stationnaire (DAS). Les sédiments dragués ont ensuite été transportés via une canalisation de refoulement et rejetés dans l'Adour (figure 1). Afin de favoriser la dispersion vers le large, les rejets ont été effectués en période de vives eaux établies et lors des courants de jusant, dans une période s'étalant d'une heure après la pleine mer $(\mathrm{PM}+1)$ à une heure avant la basse mer (BM-1), soit des périodes d'intervention de $4 \mathrm{~h}$ en continu.

Dans ce contexte, la Communauté d'Agglomération Pays Basque a mis en œuvre un Programme de Surveillance et de Suivi Environnemental (PSSE) pour chaque opération de dragage dans le but de réaliser, entre autre, un suivi continu des matières en suspension (MES) en amont et en aval de la zone de rejet.

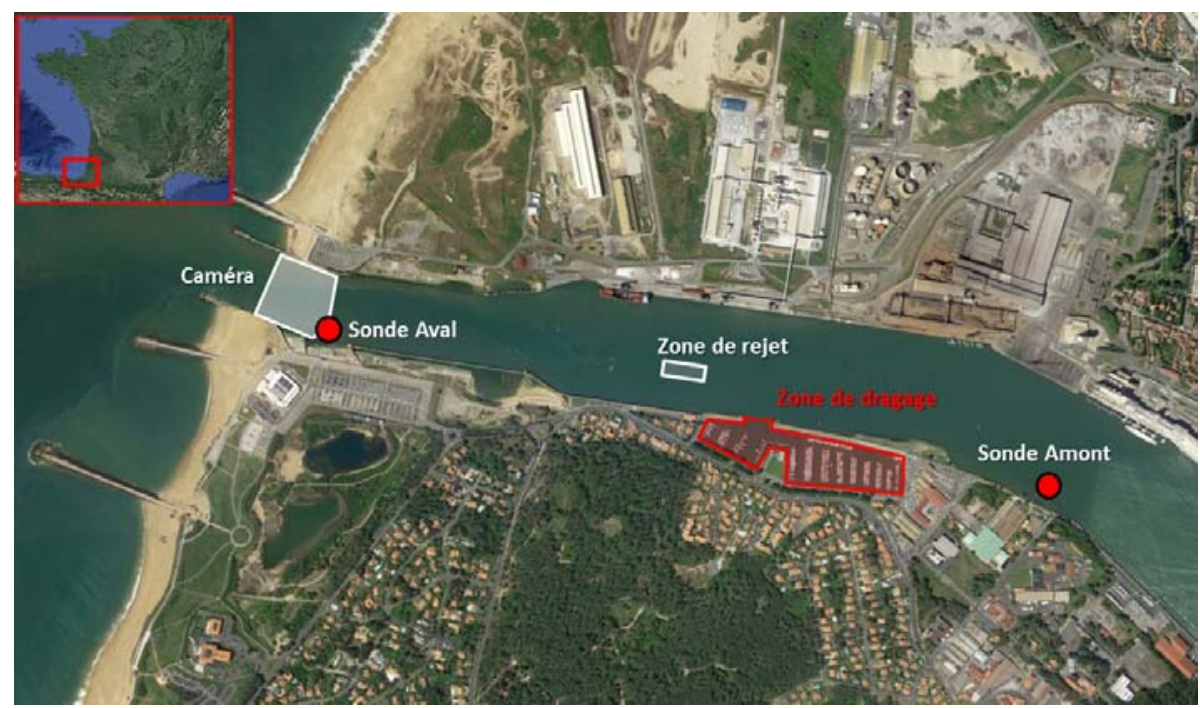

Figure 1. Localisation du site d'étude : Zone de dragage et de rejet, positions des turbidimètres aval et amont et champ de vision de la caméra.

Pour ce faire, deux turbidimètres de surface ont été installés en rive gauche entre le 15 Mars et le 15 Mai 2017, à environ $900 \mathrm{~m}$ en amont et en aval de la zone de rejet. Ces sondes permettent de suivre en temps réel les valeurs de concentration en MES via une interface Web. Parallèlement, un système de suivi vidéo a été mis en place au niveau de la tour des Signaux, à proximité de la sonde aval, pour compléter le suivi réalisé par les turbidimètres.

Cette étude porte sur l'évaluation de la capacité du système vidéo à réaliser un suivi des MES de surface au cours des activités de rejet de dragage. 


\section{XVèmes Journées Nationales Génie Côtier - Génie Civil \\ La Rochelle, 29 au 31 mai 2018}

\section{Présentation du protocole de suivi des matières en suspension - MES}

\subsection{Turbidimètre}

Le suivi de la turbidité des eaux de surface a été réalisé à partir de sondes IJINUS qui sont composées d'une bouée autonome GSM/GPRS et d'un capteur de turbidité. Leur petite taille et leur robustesse facilitent les protocoles d'installation sur site et d'entretien. Ce type de sonde permet de réaliser des mesures à une fréquence de $1 \mathrm{~Hz}$ moyennées toutes les 10 minutes. Les données sont stockées dans le datalogger embarqué puis transmises via une antenne GSM/GPRS vers un serveur FTP et une plateforme Web pour un affichage en temps réel.

\subsection{Le système vidéo}

Le système vidéo proposé par CASAGEC INGENIERIE permet, à partir d'images numériques, d'analyser et de quantifier les évolutions du littoral, et ainsi d'augmenter la qualité de la gestion de ces zones fortement dynamiques. Le système est principalement utilisé dans le cadre de diagnostics morpho-sédimentaires, de gestion proactive des opérations d'entretien de plage, et de diagnostics houle / structure / plage.

Dans le cadre de cette étude une caméra rotative a été configurée de manière à observer la zone estuarienne située au pied de la tour des Signaux de part et d'autre des rives, avec une vision la plus verticale possible. La partie du champ de vision la plus en amont est localisée à moins de $15 \mathrm{~m}$ en aval de la sonde de turbidité. Les données enregistrées à une fréquence horaire sont constituées : (i) d'une image instantanée permettant d'observer les conditions d'agitation au niveau de l'embouchure, et (ii) d'une image moyennée sur 3 minutes (images acquises à $2 \mathrm{~Hz}$ ) qui permet de lisser la surface libre et ainsi de s'affranchir des vaguelettes de surface et des éléments perturbateurs (passage de bateaux, etc.). Entre le 29 mars et le 15 avril, un ensemble de 433 images a ainsi pu être récolté.

\section{Algorithme $[\mathrm{MES}]=\mathbf{f}(\mathrm{RGB})$}

\subsection{Calibration de la sonde de turbidité aval}

Dans le but de calibrer la sonde de turbidité, 20 prélèvements d'eau ont été réalisés dans la zone estuarienne. Les échantillons d'eau ont été prélevés à l'aide d'une bouteille NISKIN et les mesures de MES ont été réalisées selon le protocole COFRAC - NF EN 872. Pour chacun des prélèvements effectués, une partie de l'eau contenue dans la bouteille NISKIN a été utilisée pour mesurer les valeurs de turbidité issues du turbidimètre. Ces valeurs de turbidité ont ensuite été comparées aux valeurs de concentration en MES pour établir les courbes d'étalonnage (figure 2). 


\section{Thème 3 - Instrumentation, mesures, imagerie et télédétection}

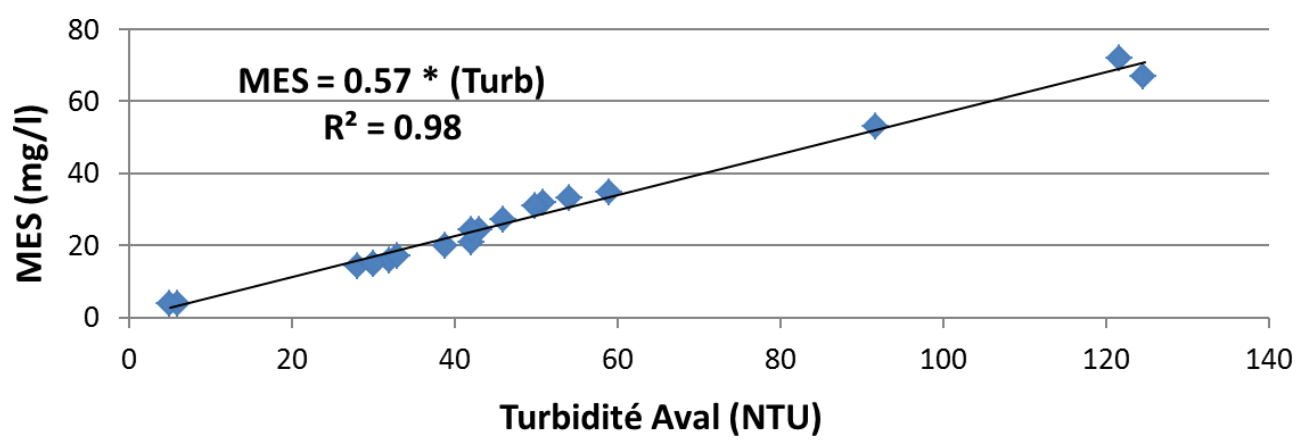

Figure 2. Courbe d'étalonnage du turbidimètre aval.

La gamme de concentration couverte au cours de cette calibration est comprise entre 3.5 et $72 \mathrm{mg} / 1$ ce qui est représentative du régime de l'Adour (PETUS, 2009). Les résultats montre une corrélation forte $\left(\mathrm{R}^{2}=0.98\right)$ entre les valeurs de turbidité enregistrées par le turbidimètre et les concentrations en MES in-situ. L'équation 1 sera donc utilisée dans la suite de l'étude pour transformer les valeurs de turbidité en valeurs de concentration en MES.

$[M E S]=0.57 \times$ Turbidité

\subsection{Calibration optique du système vidéo}

Le capteur CCD embarqué dans une caméra peut être assimilé à un capteur spectrophotométrique permettant de mesurer l'intensité lumineuse dans les longueurs d'ondes du visible (BOWERS et al., 2001 : WHITE et al., 2005 ; GODDIJN, 2007 ; GODIJJN et al., 2009). L'image numérique acquise par la caméra est une matrice constituée de 3 feuillets correspondant respectivement aux canaux rouge, vert et bleu (RVB). Les variations de concentration en MES en surface ont un impact direct sur la couleur des eaux, comme le montre la figure 3 qui présente une palette de couleur obtenue à partir de la caméra. A gauche de la palette les eaux sont bleutées donc peu chargées en MES, alors qu'elles ont une teinte marron à droite en raison d'une charge en MES plus importante.
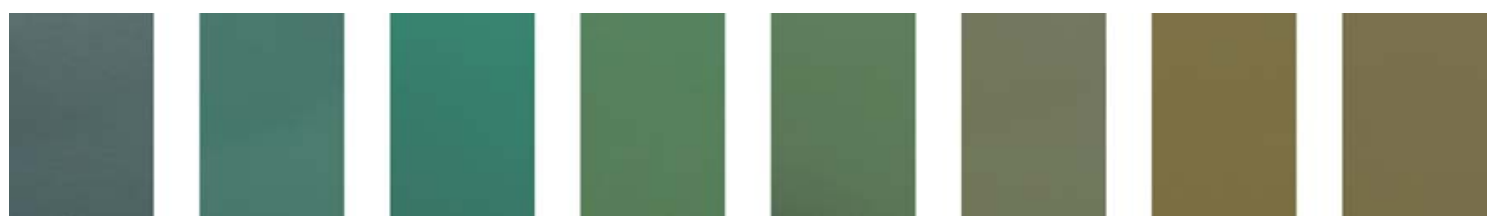

Figure 3. Exemple de palette de couleur issue des images vidéo au cours de la période de suivi.

La première étape consiste à corriger les fluctuations d'intensité lumineuse engendrées par les variations climatiques (position du soleil, couverture nuageuse, etc.). La variation d'intensité lumineuse ambiante a ainsi été corrigée à partir d'une zone étalon, 


\section{XVèmes Journées Nationales Génie Côtier - Génie Civil \\ La Rochelle, 29 au 31 mai 2018}

constituée par le sommet du pylône en premier plan. Cette zone est extraite de chaque image pour établir l'intensité RVB de la couleur blanche "vraie". Un algorithme de correction des intensités RVB est ensuite appliqué à l'ensemble de l'image. Un exemple de correction de la balance des blancs est présenté en figure 4 dans le cas d'image surexposée et sous-exposée.
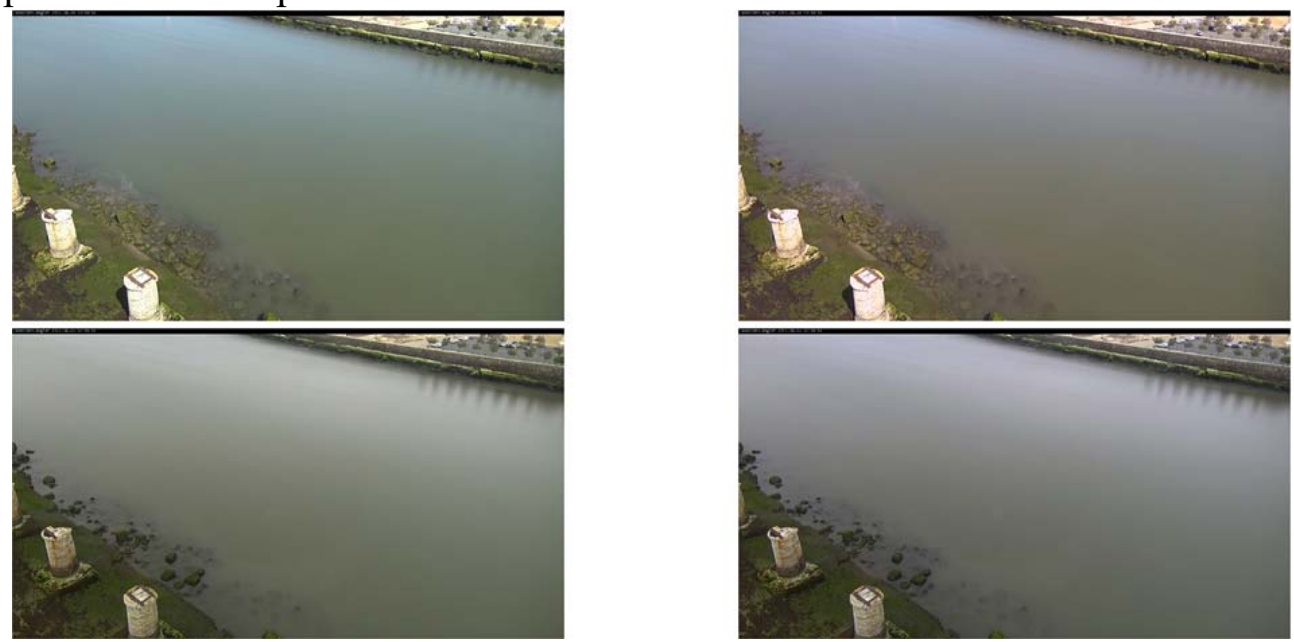

Figure 4. Exemple de correction de la balance des blancs pour une image surexposée (ligne du haut) et sous-exposée (ligne du bas) : images originales (colonne de gauche) et images corrigées (colonne de droite).

Les données RVB corrigées acquises par la caméra sont extraites à partir d'une zone d'intérêt de 150*150 pixels localisée en bas à droite de l'image afin de limiter la distance avec la sonde aval. Le code RVB est ensuite moyenné pour chacune des images.

Dans la continuité des travaux réalisés sur les caractéristiques optiques des eaux de surface du panache de l'Adour (DAILLOUX, 2008 ; PETUS et al., 2008 ; PETUS, 2009), les données de concentration en MES mesurées par la sonde aval sont confrontées au rapport de canal rouge sur vert (R/V) issu des caméras (Figure 5).

En raison de la sensibilité plus faible de la caméra, les données de concentration en MES inférieures à $7 \mathrm{mg} / 1$ ont été exclues de l'analyse. Pour le reste des données les résultats montrent une bonne corrélation $\left(\mathrm{R}^{2}=0.8\right.$, figure 5$)$ entre le rapport $\mathrm{R} / \mathrm{V}$ obtenu par la caméra et les données de MES de la sonde aval. L'algorithme obtenu est une régression polynomiale d'ordre 3 de la forme suivante :

$[M E S]=1161\left(\frac{R}{V}\right)^{3}-2505\left(\frac{R}{V}\right)^{2}+1784\left(\frac{R}{V}\right)-409$

\section{Résultats et conclusions}

L'analyse est réalisée le long d'un profil transverse à la rivière, de la rive gauche vers la rive droite. Les valeurs RVB corrigées sont récoltées sur l'intégralité du profil pour 


\section{Thème 3 - Instrumentation, mesures, imagerie et télédétection}

chaque pas de temps, puis l'algorithme (2) est appliqué au ratio R/V pour obtenir des concentrations en MES. Les données sont représentées sous forme d'un graphique (figure 6) permettant d'afficher la distance le long du profil entre la rive gauche et la rive droite (axe y), la période d'acquisition des données (axe $\mathrm{x}$ ), et les fluctuations de concentration en MES correspondantes selon un code couleur (orange : faible concentration, noir : forte concentration). Ces résultats sont confrontés aux données de la sonde aval et des débits de l'Adour.

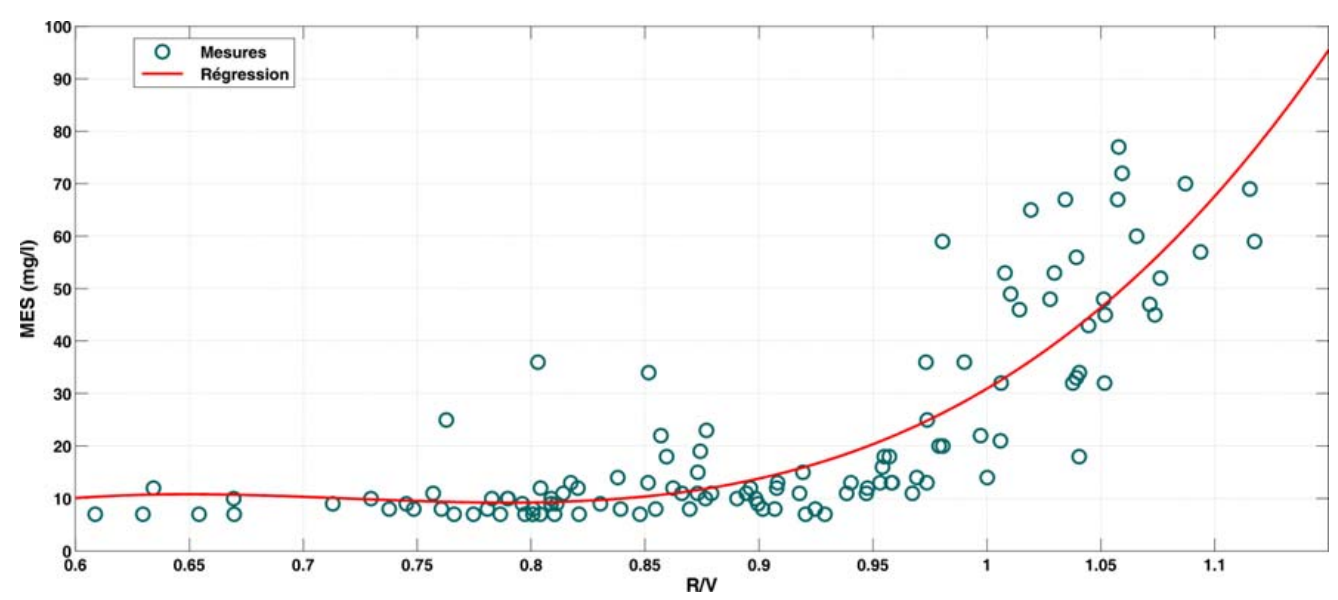

Figure 5. Variation des concentrations en MES en fonction du rapport $R / V$ corrigé.

Entre le $1^{\text {er }}$ et le 5 avril, une crue de l'Adour engendre une augmentation généralisée et continue des concentrations en MES sur l'intégralité de la section de l'estuaire. Les concentrations en MES peuvent être supérieures à $60 \mathrm{mg} / \mathrm{l}$. Ce pic de concentration est aussi observé sur les données de la sonde aval. Sur le reste de la période de suivi, l'augmentation de turbidité due aux activités de rejet est nettement visible à intervalles réguliers. Bien qu'ils soient observables périodiquement sur l'intégralité de la section, les rejets sont plus régulièrement visibles à proximité des rives droite et gauche de l'estuaire et engendrent des concentrations en MES comprises entre 25 et $45 \mathrm{mg} / \mathrm{l}$. Par ailleurs, entre le 17 et le 23 avril, les pics de concentration enregistrés par la sonde sont dus à la présence de biofouling sur le capteur de turbidité et ne sont donc pas représentatifs de la concentration in-situ (ces données ont été aussi exclues lors de la mise en place de l'algorithme (2), Section 3.2). En conséquence, aucun pic de concentration n'est observé par le système vidéo sur la même période.

Le système vidéo a permis d'améliorer la spatialisation des informations relatives à la dynamique de surface du panache turbide issu des activités de rejet. Alors que la sonde donne une information en un point de l'estuaire, la caméra a permis de mettre en avant l'écoulement des eaux turbides sur les rives droite et gauche de l'estuaire. Ce phénomène a pu être confirmé via le déploiement d'une campagne ADCP le 15 avril, qui a permis d'obtenir une information quasi 3D des écoulements entre la zone de rejet 


\section{XVèmes Journées Nationales Génie Côtier - Génie Civil \\ La Rochelle, 29 au 31 mai 2018}

et le champ de vision de la caméra. Le signal acoustique rétrodiffusé issu du courantomètre est utilisé pour obtenir une estimation de la turbidité le long de la colonne d'eau. Les résultats (figure 7) montrent que les eaux turbides au niveau du rejet se propagent rapidement sur les rives de l'estuaire et sur une couche de surface d'environ $50 \mathrm{~cm}$ à $1 \mathrm{~m}$ d'épaisseur, alors que les niveaux de turbidité sont beaucoup plus faibles au centre de l'estuaire.

Le système vidéo utilisé dans le cadre de cette étude a permis d'améliorer les connaissances en termes de propagation de surface du panache turbide issu du rejet des activités de dragage du port du Brise-Lames d'Anglet. Contrairement à la mesure en un point fixe réalisée à partir d'une sonde in-situ, la spatialisation des données est améliorée par le champ de couverture de la caméra. Par ailleurs, du fait de sa nature non-intrusive, ce type de mesure n'est pas impacté par les perturbations du milieu (biofouling, présence de déchet flottant, etc.). Néanmoins, la correction des fluctuations d'intensité lumineuse naturelle a été abordée par une approche simpliste basée sur la correction de la couleur des blancs. Des mesures spectrophotométriques des eaux de surface et une calibration du capteur CCD de la caméra doivent être envisagés pour améliorer la précision de la mesure vidéo des concentrations en MES.
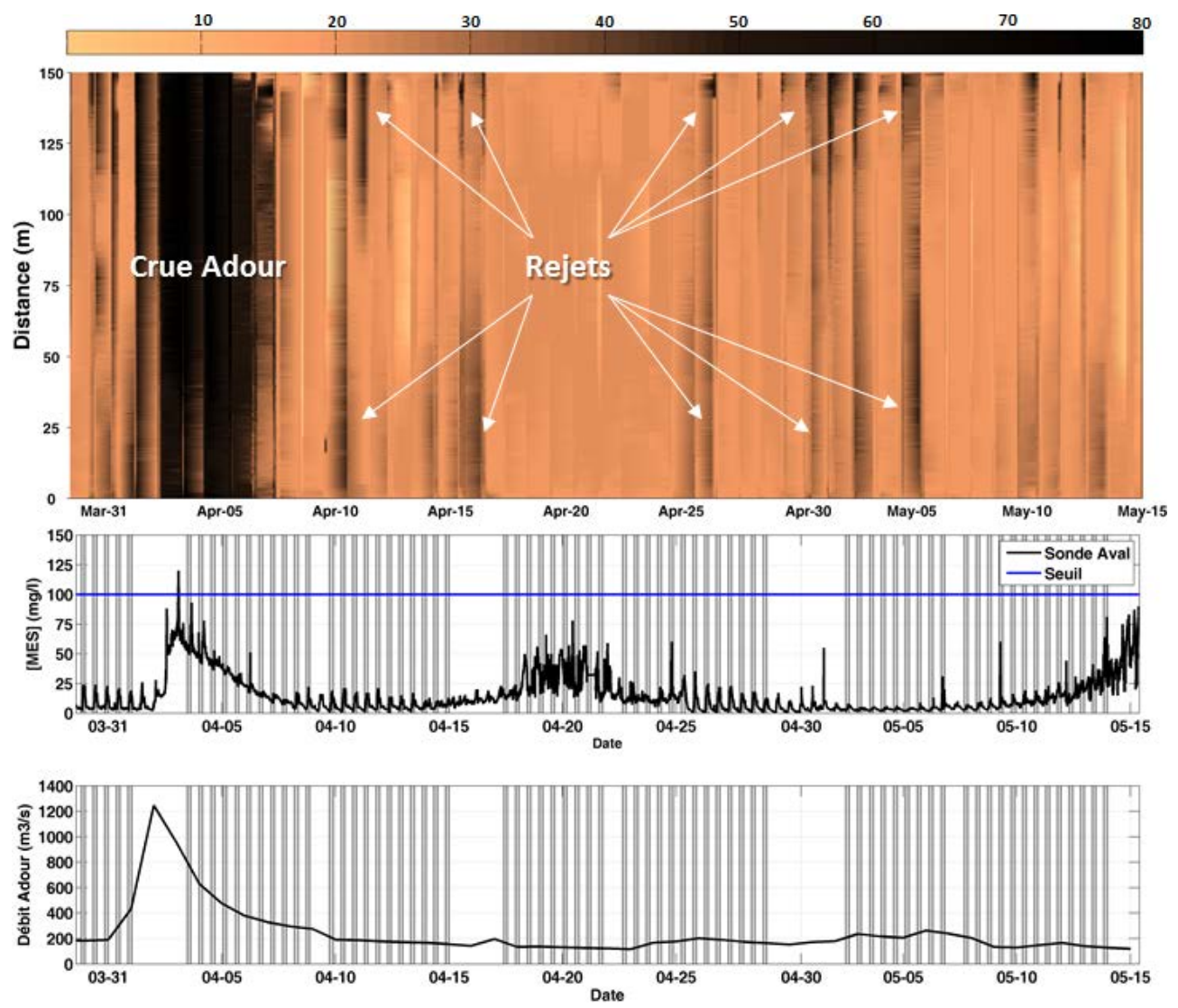

Figure 6. Variation des concentrations en MES en fonction du rapport $R / V$ issue de la caméra. 


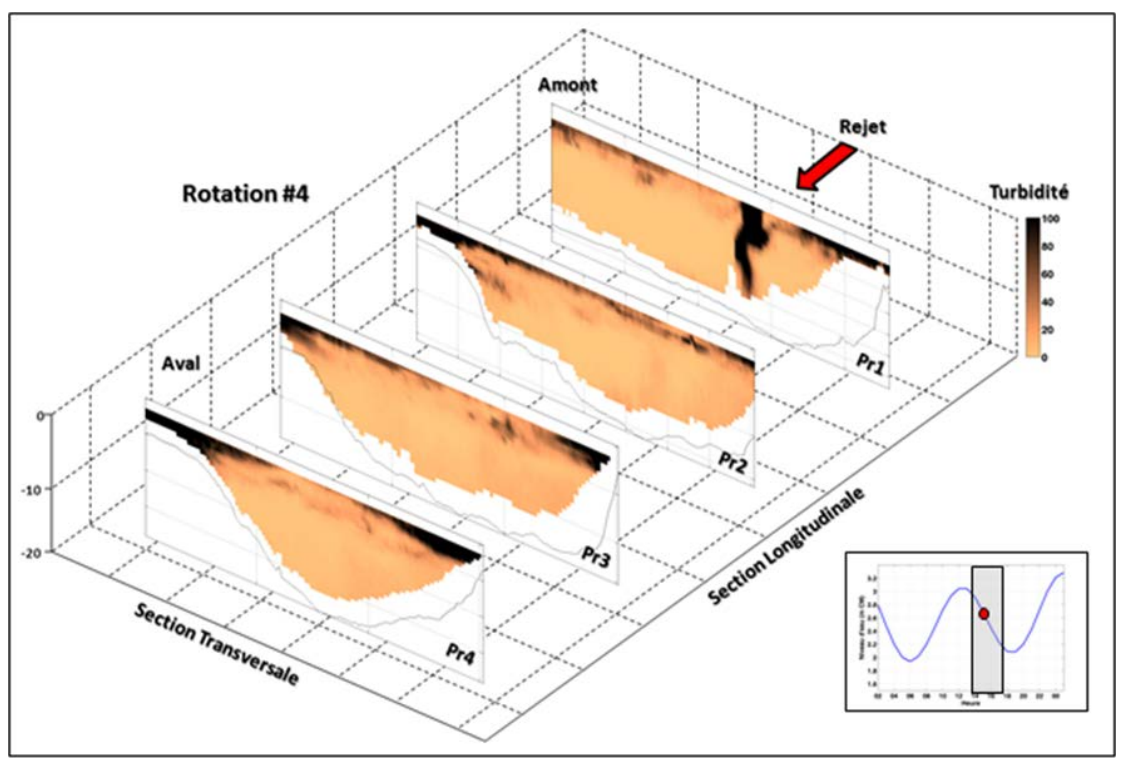

Figure 7. Exemple de suivi de la turbidité sur un cycle de mesure réalisé à partir d'un courantomètre de type ADCP le 15 avril 2017.

\section{Références bibliographiques}

BOWERS D.G., KRATZER S., MORRISON J.R., SMITH P. S.D., TETT P., WALNE A.W., WILD-ALLEN K. (2001). On the calibration and use of in situ ocean colour measurements for monitoring algal blooms. Int. J. Remote Sens, Vol. 22, pp 359-368. https://doi.org/10.1080/014311601449970

DAILlOUX D. (2008). Video measurements of the Adour plume dynamic and its surface optical characteristics. Thèse Université de Pau et des Pays de l'Adour, Anglet. GODDIJN L. M. (2007). Using a digital camera for water quality measurements in Galway bay. Ph.D. Thesis, Department of Earth and Ocean Sciences, NUIG, Galway, Ireland.

GODDIJN L. M., DAILLOUX D., WHITE M., BOWERS D. (2009). Fundamentals of in situ digital camera methodology for water quality monitoring of coast and ocean. Sensors, Vol. 9, pp 5825-5843. https://doi.org/10.3390/s90705825

PETUS C. (2009). Qualité des eaux côtières du Sud du Golfe de Gascogne par télédétection spatiale. Thèse de l'Université de Bordeaux.

PETUS C., CHUST G., SAGARMINADA Y., FROIDEFOND J. M. (2008). Estimating turbidity indicators and total suspended matter in the Bay of Biscay using MODIS 250$m$ imagery. XI International Symposium on Oceanography, San Sebastian.

WHITE M., FEIGHERY L., BOWERS D., O'RIAIN G., BOWYER P. (2005). Using a digital camera for river plume and water quality measurements. Int. J. Remote Sens. Vol. 26, pp 4405-4419. https://doi.org/10.1080/01431160500222574 